\title{
Endocytoscopy Is Useful for the Diagnosis of Superficial Nonampullary Duodenal Epithelial Tumors
}

\author{
Takashi Hirose $^{a}$ Naomi Kakushima ${ }^{a}$ Kazuhiro Furukawa ${ }^{a}$ Satoshi Furune ${ }^{a}$

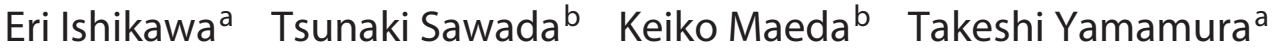 \\ Takuya Ishikawa ${ }^{a}$ Eizaburo Ohno ${ }^{a}$ Masanao Nakamura ${ }^{a} K^{2}$ Kazuki Nishida ${ }^{c}$ \\ Takio Yokoi $^{d}$ Hiroki Kawashima $^{\text {b }}$ Mitsuhiro Fujishiro ${ }^{a}$ \\ aDepartment of Gastroenterology and Hepatology, Nagoya University Graduate School of Medicine, Nagoya, Japan; \\ bDepartment of Endoscopy, Nagoya University Hospital, Nagoya, Japan; 'Biostatistics and Bioinformatics Section, \\ Center for Advanced Medicine and Clinical Research, Nagoya University Hospital, Nagoya, Japan; dDepartment of \\ Pathology and Laboratory Medicine, Nagoya University Graduate School of Medicine, Nagoya, Japan
}

\author{
Keywords \\ Endocytoscopy · Superficial nonampullary duodenal \\ epithelial tumors · Diagnosis
}

\begin{abstract}
Introduction: Endoscopic criteria for the diagnosis of superficial nonampullary duodenal epithelial tumors (SNADETs) are lacking. The aim of this study was to explore the usefulness of endocytoscopy (ECS) in the duodenum. Methods: A total of $77 \mathrm{ECS}$ images stained by methylene blue and $58 \mathrm{im}$ ages with double staining of methylene blue and crystal violet were obtained from 20 patients. Images were classified into 3 grades based on nuclear and villi atypia: EC A, B, and C. Diagnostic performance of ECS classification to predict histology and interobserver agreement was evaluated. The performance was compared between staining methods and $\times 520$ or $\times 936$ zoom. Results: With methylene blue staining, high rates of accuracy, sensitivity, specificity, and positive predictive value (PPV) over 90\% were achieved for tumor and nontumor diagnosis as assessed by EC $A$ versus EC B. High rates of accuracy, sensitivity, PPV, and negative predic-
\end{abstract}

tive value over $90 \%$ were achieved for the differentiation between the diagnosis of the Vienna category 3 and $4 / 5$ as assessed by EC B versus $C$. The accuracy rate of interpreting ECS images with $\times 936$ zoom among 10 endoscopists was $82 \%$, and the interobserver agreement rate was 0.803 (Kendall's coefficient of concordance). In the $\times 936$ zoom group, methylene blue staining was significantly associated with higher accuracy rate (odds ratio 1.76 [1.06-2.92], $p$ value 0.0297 ). No benefit was observed by double staining. Conclusions: ECS diagnosis with methylene blue provides a high accuracy rate and good interobserver agreement to predict histology of SNADETs.

(c) 2021 The Author(s) Published by S. Karger AG, Basel

\section{Introduction}

Adenocarcinoma of the small intestine is remarkably rare (incidence 6.8 cases per million) compared with other gastrointestinal carcinomas [1]. More than $50 \%$ of adenocarcinoma in the small intestine occurs in the duodenum $[2,3]$, and the incidence rate of duodenal carcinoma karger@karger.com www.karger.com/dig

Karger $\stackrel{\text { ' }}{5}$

BOPEN ACCESS
(C) 2021 The Author(s)

Published by S. Karger AG, Basel

This is an Open Access article licensed under the Creative Commons Attribution-NonCommercial-4.0 International License (CC BY-NC) (http://www.karger.com/Services/OpenAccessLicense), applicable to the online version of the article only. Usage and distribution for commercial purposes requires written permission.
Correspondence to:

Takashi Hirose, taka-h@med.nagoya-u.ac.jp

Naomi Kakushima, n.kakushima@gmail.com 


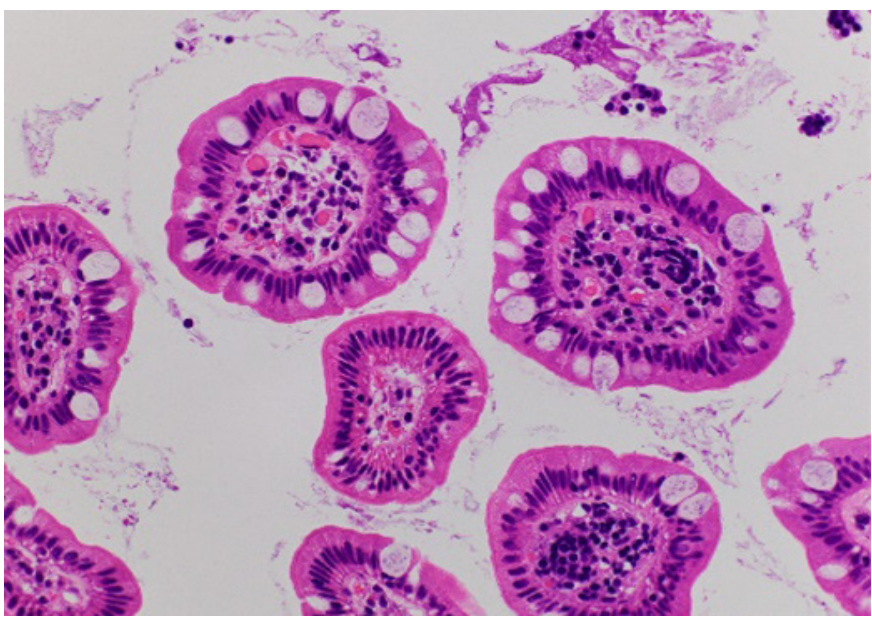

Fig. 1. Pathological image of the cut surface parallel to the mucosa of the normal duodenal mucosa.

(DC) has been reported to increase [4]. With the advance in endoscopy technology and spread of endoscopic examination, the detection of superficial nonampullary duodenal epithelial tumors (SNADETs) including adenoma or DC with invasion up to the submucosa has also increased [5-8]. Early detection and treatment are essential to achieve good prognosis for DC patients. Biopsy diagnosis is considered as a gold standard for the treatment of DC. However, the correct diagnosis rate by biopsy is not so high [9-11]. Therefore, some studies reported the utility of a classification based on white-light imaging (WLI) [6] or those using magnification with image-enhanced endoscopy (IEE) [7, 8], in order to obtain more reliable diagnosis of SNADETs. Nevertheless, universal diagnostic criteria for SNADETs have not been established yet. Furthermore, recent studies have shown good treatment results of endoscopic resection (ER) for SNADETs [12]. Biopsy-induced submucosal fibrosis sometimes makes ER much more difficult [9], and thus an endoscopic diagnosis without taking biopsies is preferred for SNADETs that are candidates for ER.

Endocytoscopy (ECS) enables the diagnosis of living tumor cells in vivo $[13,14]$ and provides a high magnification image similar to the histologic sample (Fig. 1) simply by applying the scope to the target mucosa during an endoscopic examination. It is possible to zoom in on an image with $\times 520$ magnification. In addition, ECS is equipped with an electronic zoom function which can increase the maximum magnification to $\times 936$. Although it is necessary to perform cell staining in the digestive tract, ECS has excellent image quality and image reproducibil- ity and allows the observation of nuclear variants without biopsy. In the field of gynecology, Hamou et al. [15] first attempted to observe cells using a rigid scope (Karl Storz GmbH \& Co. KG, Tuttlingen, Germany) in 1980 and reported this as "contact microscopy." In the field of gastroenterology, the efficacy of ECS in the colon has been firstly reported by Tada and Uozumi [16]. There are reports of its usefulness in the pharynx [17], esophagus [18, 19], stomach [20], and colon [21]. Although there are case reports of ECS in the duodenum [22,23], its utility is not clarified. The purpose of this study was to explore the usefulness of ECS in the duodenum and create diagnostic criteria based on ECS observation for SNADETs and consider how observation contributes to improving the accuracy rate.

\section{Materials and Methods}

\section{Eligible Patients}

This study was a retrospective analysis of a prospectively registered cohort in a university hospital. Patients with gastrointestinal neoplasm were prospectively registered to undergo diagnostic endoscopy with ECS. The cohort study using ECS was approved by the Ethics Review Board of Nagoya University (No. 2018-0290) and was registered in the University Hospital Medical Information Network (UMIN) Clinical trials (No. 000033251). Inclusion criteria for this study were patients with SNADETs that were recruited during December 2019-April 2020 and those who underwent treatment in our hospital. All of the patients had consented to the observation of ECS and participation in this study by writing. This study was conducted ethically in accordance with the World Medical Association Declaration of Helsinki update 2013.

\section{Observation with ECS}

All examinations were performed with GIF-H290EC (Olympus Medical Systems Corp., Tokyo, Japan) which has a single lens reflection, observation range of $570 \times 500 \mu \mathrm{m}$, an outer diameter of $9.7 \mathrm{~mm}$, and offers $\times 520$ magnification. All procedures for patients (conventional endoscopic and ECS observation) were performed by one experienced endoscopist (T.H.). A black silicone cap (Distal Hood MAJ-1989; Olympus Medical Systems Corp., Tokyo, Japan) was placed at the tip of the ECS diagonally. Conscious sedation was used in all cases, with intravenous injection of $5 \mathrm{mg}$ diazepam. Observation was performed after suppressing gastrointestinal peristalsis using scopolamine butylbromide or glucagon. Lesions were first washed carefully with dimethicone dissolved in water to washout mucus on the surface. In all cases, ECS was performed after white-light endoscopy and magnifying endoscopy by narrow-band imaging. The lesions were stained with $1 \%$ methylene blue. In a 5-cc syringe, 1-cc methylene blue was aspirated with 4-cc air, and a few drops were applied to the lesions by a catheter tube. Approximately 30-60 s later, excessive stain was washed out by water. The tip of the endoscope was gently contacted to the region of interest to perform ECS imaging. Careful observation was 
Table 1. ECS classification for SNADETs: EC A, B, and C according to the size and shape of nuclei, presence or absence of villi, and their architecture

\begin{tabular}{ll}
\hline Classification & Endoscopic findings \\
\hline EC A & $\begin{array}{l}\text { Small, roundish, weakly stained nuclei are evenly present. Regular villi structure is present, lined up } \\
\text { with clearly distinguishable surface epithelial cells }\end{array}$ \\
\hline EC B & $\begin{array}{l}\text { Elongated, fusiform, strongly stained nuclei are dominant, but the orientation of the nuclei is relatively } \\
\text { maintained. Villi structure is obscure with diminished visualization of the surface epithelium }\end{array}$ \\
\hline EC C & $\begin{array}{l}\text { Large, irregular nuclei are dominant with various degrees of staining. Villi structure is absent, and a } \\
\text { crypt orifice with increased number of cells around the crypt is observed }\end{array}$ \\
\hline
\end{tabular}

SNADETs, superficial nonampullary duodenal epithelial tumors; ECS, endocytoscopy.

carried out to prevent bleeding due to excessive pressure. Images with $\times 520$ and $\times 936$ magnification were obtained for the lesions and surrounding normal villi. Additional ECS observation was performed by double staining with $1 \%$ methylene blue and $0.05 \%$ crystal violet.

\section{Development of ECS Classification}

On the basis of the results of ECS in the colon [21], the endoscopic images obtained by ECS were classified into 3 grades: EC A, $\mathrm{B}$, and $\mathrm{C}$ according to the size and shape of nuclei, presence or $\mathrm{ab}$ sence of villi, and their architecture (Table 1).

- EC A: small, roundish, weakly stained nuclei are evenly present. Regular villi structure is present, lined up with clearly distinguishable surface epithelial cells (Fig. 2a: image with methylene blue staining and Fig. 2b: double staining).

- EC B: elongated, fusiform, strongly stained nuclei are dominant, but the orientation of the nuclei is relatively maintained. Villi structure is obscure with diminished visualization of the surface epithelium (Fig. 2c: image with methylene blue staining and Fig. 2d: double staining).

- EC C: large, irregular nuclei are dominant with various degrees of staining. Villi structure is absent, and a crypt orifice with increased number of cells around the crypt is observed (Fig. 2e: image with methylene blue staining and Fig. 2f: double staining).

EC A was considered as Vienna classification category 1 (C1), EC B was considered as category 3 (C3), and EC C was considered as category 4 or $5(\mathrm{C} 4 / 5)$ [24]. Histological assessment of resected specimens was based on the Vienna classification by multiple pathologists. When the lesion had histological heterogeneity, careful mapping was performed regarding the pathological diagnosis of each observation area. The border of the lesions was identified during endoscopic observation, and areas of inner part (lesion) and outer part (nonlesion) of the proximal border were observed with ECS. The resected specimen was fixed with the indication of the proximal border. The EC A image was also consistent with the previous report [25].

\section{Interpretation of ECS Images}

Magnified endoscopic images stained with methylene blue which included normal villi, SNADETs with $\times 520$, and electronic zoom magnification $(\times 936)$ were selected. When selecting multiple images from one case, we selected images focusing on different parts of the lesion.

First, an expert endoscopist (T.H.) evaluated and examined the validity of ECS classification using the selected images. At the time of ECS diagnosis and evaluation, patient information and images of white-light and NBI endoscopic images were blinded, and the evaluation was performed by using only ECS observation images. The accuracy rate, sensitivity, specificity, positive predictive value (PPV), and negative predictive value (NPV) were calculated for the diagnosis of tumor or nontumor and Vienna classification C3 or C4/5 for the SNADETs (step 1).

Next, 10 board certified endoscopists who did not know clinical information and different from the operator evaluated similar ECS images based on the new classification. The ECS images were randomly presented to the observers who were blinded to both WLI or IEE images and histology. The concordance rate of the diagnosis and the diagnostic performance were evaluated (step 2). Finally, staining methods and levels of magnification were statistically evaluated to extract the best condition contributing to the improvement of the accuracy rate of ECS classification (step 3).

\section{Statistical Analysis}

For patient characteristics, categorical variables were expressed as percentages, and continuous variables were summarized using median and interquartile range (IQR), respectively. Consistency among 10 readers was examined using Kendall's coefficient of concordance. As for the main analysis, the effects of staining and magnification on the improvement of the correct answer rate were performed by using the generalized linear mixed effect model. First, we examined the interaction between staining and magnification and then examined the effect of staining by stratifying the presence or absence of magnification. Throughout all models, differences between interpreters were treated as random effects, and the magnitude of staining, magnification, and interaction between staining and magnification was assessed by fixed effects. $p$ value $<0.05$ was considered statistically significant. All statistical analyses were performed using R version 3.6.1 and SPSS version 26.0.0 software program (IBM Corp., Armonk, NY, USA). 


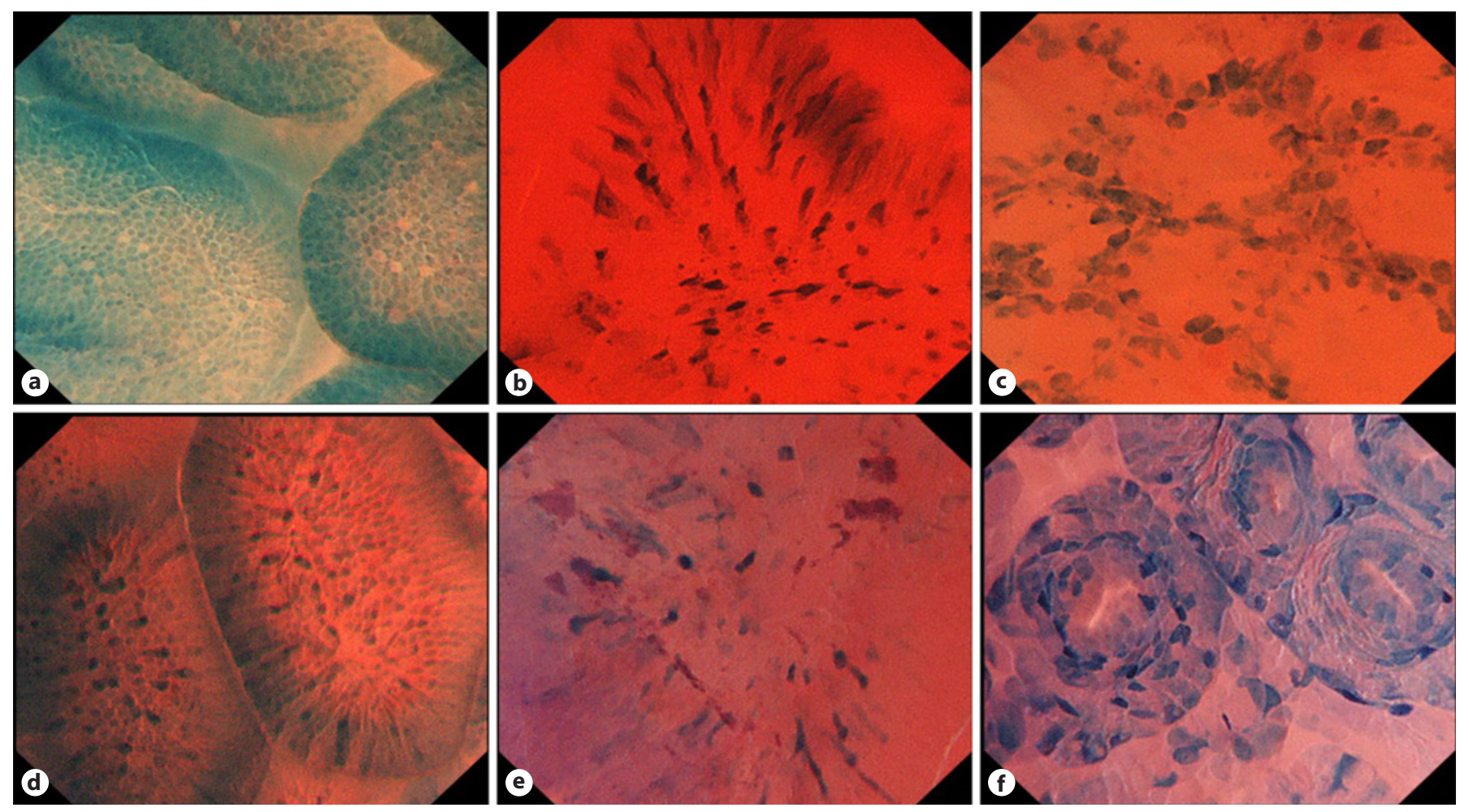

Fig. 2. a EC A image with methylene blue staining: small, roundish, weakly stained nuclei are evenly present. Regular villi structure is present, lined up with clearly distinguishable surface epithelial cells $(\times 936)$. b EC A image with double staining $(\times 936)$. c EC B image with methylene blue staining: elongated, fusiform, strongly stained nuclei are dominant, but the orientation of the nuclei is relatively maintained. Villi structure is obscure with diminished

\section{Results}

A total of 20 (14 males and 6 females) patients underwent ECS during the study period and received subsequent ER. Thorough pathological evaluation was carried out using en bloc resection specimens in all cases. Patient and lesion characteristics are shown in Table 2. A total of 77 images stained by methylene blue ( 26 of $\times 520$ images and 51 electronic zoom $\times 936$ images) were selected from 20 cases. Images for double staining included a total of 58 images ( 24 of $\times 520$ images and 34 of $\times 936$ images $)$ selected from 14 cases. Images were evenly obtained from each case; 1 normal villi image $(\times 520$ or $\times 936), 1$ or 2 $\times 520$ lesion images, and 1 or $2 \times 936$ lesion images. Clear ECS images were obtained in all cases. The median time of staining and observation was 6 min (range, 5-9) by methylene blue staining and 6 min (range, 4-6) by double staining. The mean examination time was $30 \mathrm{~min}$ (range, 25-40) for a complete upper gastrointestinal en- visualization of the surface epithelium $(\times 936)$. d EC B image with double staining ( $\times 936)$. e EC C image with methylene blue staining: large, irregular nuclei are dominant with various degrees of staining. Villi structure is absent, and a crypt orifice with increased number of cells around the crypt is observed (×936). $\mathbf{f}$ EC C image with double staining $(\times 936)$.

doscopy, including examination of the esophagus and stomach. No adverse events were noted during this procedure.

\section{Diagnostic Performance of ECS Classification by a}

Single Expert (T.H.) (Step 1)

The diagnostic performance of the new ECS classification assessed by images with methylene blue staining or double staining is shown in Table 3. With methylene blue staining, high rates of accuracy, sensitivity, specificity, and PPV over $90 \%$ were achieved for nontumor and tumor diagnosis as assessed by EC A versus EC B and C (Table 4). High rates of accuracy, sensitivity, PPV, and NPV over $90 \%$ were achieved for the differentiation between the diagnosis of $\mathrm{C} 3$ and $\mathrm{C} 4 / 5$ as assessed by EC B versus $C$. With double staining, similar results were observed for nontumor and tumor diagnosis; however, the diagnostic performance was rather lower for the differentiation between C3 and C4/5 (Table 4). 
Table 2. Patient and lesion characteristics who underwent ECS for SNADETs

\begin{tabular}{lc}
\hline Macroscope type, $n(\%)$ & \\
$0-\mathrm{I}$ & $3(15)$ \\
0-IIa & $10(50)$ \\
0-IIc & $7(35)$ \\
Size, median (range), mm & $14(13-21)$ \\
\hline Treatment, $n$ (\%) & \\
Endoscopic submucosal dissection & $13(65)$ \\
Endoscopic mucosal resection & $6(30)$ \\
Cold snare polypectomy & $1(5)$ \\
\hline Histology, $n(\%)$ & \\
C3 345 & $7(35)$ \\
C45 & $13(65)$ \\
\hline
\end{tabular}

SNADETs, superficial nonampullary duodenal epithelial tumors; ECS, endocytoscopy.
Table 3. Correlation between ECS diagnosis and histopathological diagnosis with methylene blue staining or double staining

\begin{tabular}{lrrrr}
\hline & \multicolumn{2}{c}{ Vienna classification } & Total \\
\cline { 2 - 3 } & C1 & C3 & C4-5 & \\
\hline EC with MB & & & & \\
A & 17 & 3 & 0 & 20 \\
B & 1 & 13 & 0 & 14 \\
C & 0 & 4 & 39 & 43 \\
\hline EC with DS & 12 & 0 & 0 & 12 \\
A & 1 & 9 & 0 & 10 \\
B & 0 & 5 & 31 & 36 \\
C & & & & \\
\hline
\end{tabular}

ECS, endocytoscopy; MB, methylene blue; DS, double staining.

Table 4. The diagnostic performance of ECS with methylene blue staining or double staining

\begin{tabular}{|c|c|c|c|c|}
\hline & \multicolumn{2}{|c|}{ Tumor or nontumor } & \multicolumn{2}{|l|}{$\mathrm{C} 3$ or $\mathrm{C} 4 / 5$} \\
\hline & $\mathrm{MB}$ & DS & $\mathrm{MB}$ & DS \\
\hline Accuracy & $\begin{array}{l}94.80 \% \\
(87.2-98.6)\end{array}$ & $\begin{array}{l}98.30 \% \\
(90.8-100)\end{array}$ & $\begin{array}{l}92.90 \% \\
(82.7-98.0)\end{array}$ & $\begin{array}{l}88.90 \% \\
(75.9-96.3)\end{array}$ \\
\hline Sensitivity & $\begin{array}{l}94.90 \% \\
(85.9-98.9)\end{array}$ & $\begin{array}{l}100 \% \\
(92.1-100)\end{array}$ & $\begin{array}{l}100 \% \\
(91.0-100)\end{array}$ & $\begin{array}{l}100 \% \\
(88.8-100)\end{array}$ \\
\hline Specificity & $\begin{array}{l}94.40 \% \\
(72.7-99.9)\end{array}$ & $\begin{array}{l}92.30 \% \\
(64.0-99.8)\end{array}$ & $\begin{array}{l}76.50 \% \\
(50.1-93.2)\end{array}$ & $\begin{array}{l}64.30 \% \\
(35.1-87.2)\end{array}$ \\
\hline PPV & $\begin{array}{l}98.20 \% \\
(90.6-100)\end{array}$ & $\begin{array}{l}97.80 \% \\
(88.5-99.9)\end{array}$ & $\begin{array}{l}90.70 \% \\
(77.9-97.4)\end{array}$ & $\begin{array}{l}86.10 \% \\
(70.5-95.3)\end{array}$ \\
\hline NPV & $\begin{array}{l}85.00 \% \\
(62.1-96.8)\end{array}$ & $\begin{array}{l}100 \% \\
(73.5-100)\end{array}$ & $\begin{array}{l}100 \% \\
(75.3-100)\end{array}$ & $\begin{array}{l}100 \% \\
(66.4-100)\end{array}$ \\
\hline
\end{tabular}

ECS, endocytoscopy; PPV, positive predictive value; NPV, negative predictive value; MB, methylene blue; DS, double staining.

\section{Interobserver Interpretation of ECS Classification}

(Step 2)

The results of 10 endoscopists are shown in Table 5. By methylene blue staining, the accuracy rate of interpreting the images with electronic zoom was $82.4 \%$ and of the images without electronic zoom was 63.5\%. Diagnostic agreement rates for images with and without electronic zoom (Kendall's coefficient of concordance) in 10 interpreters were 0.803 and 0.730 , respectively (Table 5 ). For each classification, the accuracy rates with electronic zoom in 10 interpreters were $89.3 \%$ (range, 76.8-92.9) in EC A, 75.0\% (range, 66.7-91.7) in EC B, and 82.0\% (range, 78.0-94.0) in EC C, respectively. There was no significant difference in the accuracy rate between EC A, B, and C $(p=0.165)$.

By double staining, the accuracy rates of the images with electronic zoom were $72.1 \%$ and images without electronic zoom were $66.7 \%$. Diagnostic agreement rates for images with and without electronic zoom (Kendall's coefficient of concordance) in 10 interpreters were 0.739 and 0.661 , respectively (Table 5). The accuracy rates with electronic zoom were $88.9 \%$ (range, 63.9-88.9) in EC A, $62.5 \%$ (range, 62.5-75.0) in EC B, and 79.4\% (range, 58.888.2) in EC C, respectively. There was no significant difference in the accuracy rate between EC A, B, and C ( $p=$ 0.082 ). 
Table 5. Comparison of accuracy and diagnostic agreement rate for ECS diagnosis

\begin{tabular}{|c|c|c|c|c|}
\hline & Methylene & le staining & Double stair & \\
\hline & electronic $\mathrm{z}$ & & electronic $\mathrm{zc}$ & \\
\hline & $(-)$ & $(+)$ & $(-)$ & $(+)$ \\
\hline Accuracy rate & $\begin{array}{l}63.50 \% \\
(60.6-71.1)\end{array}$ & $\begin{array}{l}82.40 \% \\
(77.9-87.3)\end{array}$ & $\begin{array}{l}66.70 \% \\
(57.3-87.5)\end{array}$ & $\begin{array}{l}72.10 \% \\
(70.6-82.4)\end{array}$ \\
\hline Diagnostic agreement rate & 0.73 & 0.803 & 0.661 & 0.739 \\
\hline
\end{tabular}

ECS, endocytoscopy.
Best Condition Contributing to the Improvement of the Accuracy Rate of ECS Classification (Step 3)

In step 3, all analyses were performed using linear mixed-effects models. First, when we applied the model by examining the effect of methylene blue staining on the accuracy rate of ECS with adjustment of magnification, no statistically significant difference was obtained (odds ratio 1.27 [0.99-1.63], $p=0.063$ ). However, when the interaction term between staining and magnification was added to the model above, it became clear that there was a statistically significant interaction (odds ratio 1.76 [1.06-2.92], $p=0.0297$ ).

Since an interaction was observed, subgroup analysis was performed in the electronic zoom group and the $\times 520$ observation group. In the electronic zoom group, methylene blue staining was significantly better in the accuracy rate as compared with double staining (odds ratio 1.55 [1.11-2.18], $p=0.011)$. On the other hand, there was no significant difference in the accuracy rate between methylene blue staining and double staining in the $\times 520$ observation group (odds ratio 0.89 [0.61-1.29], $p=0.531)$.

\section{Discussion/Conclusion}

In the present study, we evaluated the usefulness of ECS in the duodenum and created a new classification of ECS for the diagnosis of SNADETs. Our new classification was effective for the diagnosis of tumor or nontumor discrimination and also for the differentiation between $\mathrm{C} 3$ and $\mathrm{C} 4 / 5$ lesions. Both methylene blue staining and double staining showed a good diagnostic performance. Our study also showed that methylene blue staining was better than double staining in the sensitivity, specificity, accuracy rate, PPV, and NPV in distinguishing $\mathrm{C} 3$ and $\mathrm{C} 4 / 5$.

The diagnostic accuracy in 10 endoscopists was $63.5 \%$ without electron zoom and increased to $82.4 \%$ with electronic zoom. The initial analysis was done by a single ex- pert so that there may be a learning curve. Another possible reason may be the difference in which part of the EC image was of interest among the observers. But, excellent concordance (82.35\%) was found between ECS by methylene blue staining with electronic zoom and histology. Moreover, the interobserver diagnostic agreement rate was 0.803 , which was excellent. Similar to ECS studies in other gastrointestinal tract organs $[19,21]$, high accuracy rate and diagnostic agreement were obtained using ECS for duodenal villi and SNADETs. Regarding other diagnostic modalities, the accuracy rate for white-light observation was $86 \%$ [6] and that for NBI observation was $72-87 \%[8,26]$. The results of this study were comparable to those reports. Therefore, optical diagnoses including ECS could be considered as an alternative to biopsy-based diagnosis, where accuracy has been reported as 68-74\% [9-11].

The accuracy rate of EC B was slightly lower than that of EC A and C, although there was no significant difference. One possible reason may be the heterogeneity of tumors. Tumors classified as $\mathrm{C} 4$ may have areas with less atypia. All lesions were retrieved en bloc and were carefully orientated to obtain the histology observed by ECS. However, a slight deviation may still have occurred. Since this study assessed the accuracy of each individual image, obtaining several images from the lesion will help diagnose the most advanced histology in real practice.

Electronic zoom contributed to the improvement of the accuracy rate in ECS using methylene blue staining (odds ratio 1.76 [1.06-2.92], $p=0.0297$ ). Electronic zoom with $\times 936$ magnification clearly showed the enlargement and irregularity of cell nuclei, facilitating the diagnosis. The utility of electronic zoom $(\times 600)$ over standard magnification $(\times 380)$ using a prototype ECS has also been reported for lesions in the esophagus [27]. Electronic zoom would be effective especially for tumors with less glandular atypia by providing additional information of cytological atypia.

In this study, the significance of using crystal violet staining for ECS was not found. The feasibility of double 
staining with methylene blue and crystal violet in the stomach was previously reported with a good diagnostic performance [20]. In the duodenum, a previous study compared methylene blue, toluidine blue, and crystal violet and concluded that $1 \%$ methylene blue was the optimal staining condition for in vivo ECS of SNADETs [25]. Our results also showed that $1 \%$ methylene blue staining provided clear images of normal duodenal villi and SNADETs by ECS. Subgroup analysis showed that methylene blue staining significantly improved the accuracy rate in the group that underwent electronic zoom (odds ratio 1.55 [1.11-2.18], $p=0.011)$. We considered that it was sometimes difficult to distinguish between the nucleus stained with methylene blue and the cytoplasm stained with crystal violet when the number of cells were increased and nuclei were enlarged. As for crystal violet, the report of carcinogenicity to rats was mentioned by gentian violet $[28,29]$. The amount of crystal violet of ECS is very limited, so its carcinogenicity is not clear. However, since there was no clear advantage from the results of this study, we consider that the use of crystal violet is not recommended in terms of diagnosis for SNADETs.

In this study, the number of evaluated images was not the same between different staining methods and images with or without electronic zoom. Therefore, we used a generalized linear mixed effect model in statistical analysis, and there was no selection bias because of performing subgroup analysis for the condition where the interaction was obtained.

This study has some limitations. First, this study was a single-center study with limited number of cases. All of our cases were prospectively registered for ECS evaluation. Considering the rarity of SNADETs, we believe that we accumulated enough ECS images to validate our new ECS classification. Second, double staining was performed after methylene blue staining. Therefore, there may be a possibility that the image quality of the double staining was affected by previous observation. Since the diagnostic performance of methylene blue staining was satisfactory, we do not recommend double staining for ECS in the duodenum considering its labor and time. Third, one expert endoscopist performed the ECS observation, and there may be a bias that the endoscopist might have tried to record a preferable image. However, there is still no standard criteria of WLI or IEE diagnosis for SNADETs, and the final histology was not known during ECS observation. In addition, in step 2 of this study, the observers were different from the endoscopist who performed ECS observation and were blinded to WLI or IEE images. Nevertheless, interobserver agreement of the interpretation of ECS images was high among 10 endoscopists blinded to the histology. Forth, the diagnostic performance of ECS was not compared to conventional WLI or other IEE such as narrowband imaging. In general practice, neoplasms are usually detected by WLI and subsequently diagnosed by conventional methods. In the future, we should evaluate the effect of adding ECS observation to white-light and IEE observation. Finally, we did not consider the mucin phenotypes of SNADETs in this study. It is known that gastric-type SNADETs differ from intestinal-type in terms of tumor location, morphology, and biology [30]. Further studies are required whether the diagnostic ability of ECS is affected by the mucin phenotype. In conclusion, ECS diagnosis with methylene blue provides a high accuracy rate and good interobserver agreement for the diagnosis of tumor or nontumor discrimination and also for the differentiation between C3 and C4/5 lesions among SNADETs.

\section{Statement of Ethics}

The cohort study using ECS was approved by the Ethics Review Board of Nagoya University (No. 2018-0290) and was registered in the University Hospital Medical Information Network (UMIN) Clinical trials (No. 000033251). All of the patients had consented to the observation of ECS and participation in this study by writing. This study was conducted ethically in accordance with the World Medical Association Declaration of Helsinki update 2013.

\section{Conflict of Interest Statement}

The authors have no conflicts of interest to declare.

\section{Funding Sources}

GIF-H290EC was provided by Olympus Medical Systems Corp., Tokyo, Japan. However, Olympus Medical Systems did not involve in study design, recruitment of patients, study analysis, and writing of the manuscript.

\section{Author Contributions}

Conception and design: Hirose T., Kakushima N., Furukawa K., and Furune S. Analysis and interpretation of data: Hirose T., Ishikawa I., Sawada T., Maeda K., Yamamura T., Ishikawa T., Ohno E., Nakamura M., and Nishida K. Drafting of the article: Hirose T., Yokoi T., and Kawashima H. Critical revision of the article for important intellectual content: Fujishiro M. Final approval of the article: all authors. 


\section{References}

1 Schottenfeld D, Beebe-Dimmer JL, Vigneau FD. The epidemiology and pathogenesis of neoplasia in the small intestine. Ann Epidemiol. 2009; 19:58-69.

2 Dabaja BS, Suki D, Pro B, Bonnen M, Ajani J. Adenocarcinoma of the small bowel: presentation, prognostic factors, and outcome of 217 patients. Cancer. 2004;101:518-26.

3 Aparicio T, Zaanan A, Mary F, Afchain P, Manfredi S, Evans Jeffry RT. Small bowel adenocarcinoma. Gastroenterol Clin North Am. 2016;45:447-57.

4 Chow JS, Chen CC, Ahsan H, Neugut AI. A population-based study of the incidence of malignant small bowel tumors: SEER, 19731990. Int J Epidemiol. 1996;25:722-8.

5 Tsuji S, Doyama H, Tsuji K, Tsuyama S, Tominaga K, Yoshida N, et al. Preoperative endoscopic diagnosis of superficial non-ampullary duodenal epithelial tumors, including magnifying endoscopy. World J Gastroenterol. 2015;21:11832-41.

6 Kakushima N, Yoshida M, Iwai T, Kawata N, Tanaka M, Takizawa K, et al. A simple endoscopic scoring system to differentiate between duodenal adenoma and carcinoma. Endosc Int Open. 2017;5:E763-8.

7 Kikuchi D, Hoteya S, Iizuka T, Kimura R, Kaise M. Diagnostic algorithm of magnifying endoscopy with narrow band imaging for superficial non-ampullary duodenal epithelial tumors. Dig Endosc. 2014;26(Suppl 2):16-22.

8 Kakushima N, Yoshida M, Yamaguchi Y, Takizawa K, Kawata N, Tanaka M, et al. Magnified endoscopy with narrow-band imaging for the differential diagnosis of superficial non-ampullary duodenal epithelial tumors. Scand J Gastroenterol. 2019;54:128-34.

9 Kinoshita S, Nishizawa T, Ochiai Y, Uraoka T, Akimoto T, Fujimoto A, et al. Accuracy of biopsy for the preoperative diagnosis of superficial nonampullary duodenal adenocarcinoma. Gastrointest Endosc. 2017;86:329-32.

10 Goda K, Kikuchi D, Yamamoto Y, Takimoto K, Kakushima N, Morita Y, et al. Endoscopic diagnosis of superficial non-ampullary duodenal tumors in Japan: multicenter case series. Dig Endosc. 2014;26:23-9.
11 Kakushima N, Kanemoto H, Sasaki K, Kawata N, Tanaka M, Takizawa K, et al. Endoscopic and biopsy diagnoses of superficial, nonampullary, duodenal adenocarcinomas. World J Gastroenterol. 2015;21:5560-7.

12 Yahagi N, Kato M, Ochiai Y, Maehata T, Sasaki M, Kiguchi Y, et al. Outcomes of endoscopic resection for superficial duodenal epithelial neoplasia. Gastrointest Endosc. 2018; 88:676-82.

13 Inoue H, Kazawa T, Sato Y, Satodate H, Sasajima K, Kudo SE, et al. In vivo observation of living cancer cells in the esophagus, stomach, and colon using catheter-type contact endoscope, "Endo-Cytoscopy system". Gastrointest Endosc Clin N Am. 2004;14:589-94.

14 Sasajima K, Kudo SE, Inoue H, Takeuchi T, Kashida H, Hidaka E, et al. Real-time in vivo virtual histology of colorectal lesions when using the endocytoscopy system. Gastrointest Endosc. 2006;63:1010-7.

15 Hamou J, Salat-Baroux J, Coupez F, Brux JD. Microhysteroscopy: a new approach to the diagnosis of cervical intraepithelial neoplasia. Obstet Gynecol. 1984;63:567-74.

16 Tada M, Uozumi Y. A new method for the ultra-magnifying observation of the colon mucosa. Kyoto Prefect Univ Med. 1982;91: 349-54.

17 Shimizu Y, Takahashi M, Yoshida T, Ono S, Mabe K, Kato M, et al. Endoscopic in vivo cellular imaging of superficial squamous cell carcinoma of the head and neck by using an integrated endocytoscopy system (with video). Gastrointest Endosc. 2013;78:351-8.

18 Kumagai Y, Monma K, Kawada K. Magnifying chromoendoscopy of the esophagus: invivo pathological diagnosis using an endocytoscopy system. Endoscopy. 2004;36:590-4.

19 Inoue H, Sasajima K, Kaga M, Sugaya S, Sato $\mathrm{Y}$, Wada Y, et al. Endoscopic in vivo evaluation of tissue atypia in the esophagus using a newly designed integrated endocytoscopy: a pilot trial. Endoscopy. 2006;38:891-5.

20 Kaise M, Ohkura Y, Iizuka T, Kimura R, Nomura K, Kuribayashi Y, et al. Endocytoscopy is a promising modality with high diagnostic accuracy for gastric cancer. Endoscopy. 2015; 47:19-25.
21 Kudo S-E, Wakamura K, Ikehara N, Mori Y, Inoue $\mathrm{H}$, Hamatani S. Diagnosis of colorectal lesions with a novel endocytoscopic classification: a pilot study. Endoscopy. 2011;43:86975.

22 Miyamoto S, Kudo T, Abiko S, Ono S, Shimizu Y, et al. Endocytoscopy of superficial nonampullary duodenal epithelial tumor: two cases of tubular adenocarcinoma and adenoma. Am J Gastroenterol. 2017;112:1638.

23 Kumagai Y, Higashi M, Muramatsu S, Mochiki E, Ishida H. Endocytoscopic observation of non-ampullary mucosal duodenal cancer. Case Rep Gastroenterol. 2020;14:15664.

24 Schlemper RJ, Riddell RH, Kato Y, Borchard F, Cooper HS, Dawsey SM, et al. The vienna classification of gastrointestinal epithelial neoplasia. Gut. 2000;47:251-5.

25 Goda K, Dobashi A, Yoshimura N, Hara Y, Tamai N, Sumiyama K, et al. Dye solution optimizing staining conditions for in vivo endocytoscopy for normal villi and superficial epithelial tumors in the duodenum. Ann Gastroenterol. 2019;32:378-86.

26 Mizumoto T, Sanomura Y, Tanaka S, Kuroki K, Kurihara M, Yoshifuku Y, et al. Clinical usefulness of magnifying endoscopy for nonampullary duodenal tumors. Endosc Int Open. 2017;5:E297-302.

27 Kumagai $Y$, Kawada K, Higashi T, Ishiguro T, Sobajima J, Fukuchi M, et al. Endocytoscopic observation of various esophageal lesions at $\times 600$ : can nuclear abnormality be recognized? Dis Esophagus. 2015;28:269-75.

28 Littlefield NA, Gaylor DW, Blackwell BN, Allen RR. Chronic toxicity/carcinogenicity studies of gentian violet in Fischer 344 rats: two-generation exposure. Food Chem Toxicol. 1989;27:239-47.

29 Littlefield NA, Blackwell B-N, Hewitt CC, Gaylor DW. Chronic toxicity and carcinogenicity studies of gentian violet in mice. Fundam Appl Toxicol. 1985;5:902-12.

30 Yoshida M, Shimoda T, Abe M, Kakushima N, Kawata N, Takizawa K, et al. Clinicopathological characteristics of non-ampullary duodenal tumors and their phenotypic classification. Pathol Int. 2019;69:398-406. 